\title{
Limiting research on molecular targeted therapies in advanced solid cancers: beyond a cost-effectiveness ratio analysis
}

\author{
Andrea Nicolini ${ }^{*, 1}$, Paola Ferrari ${ }^{1} \&$ Angelo Carpi $^{2}$ \\ ${ }^{1}$ Department of Oncology, Transplantations \& New Technologies in Medicine, University of Pisa, Italy \\ ${ }^{2}$ Department of Clinical \& Experimental Medicine, University of Pisa, Italy \\ *Author for correspondence: Tel.: +39 339872 4215; andrea.nicolini@med.unipi.it
}

\begin{abstract}
'The lack of effectiveness in most unselected patients, and high treatment costs make the inclusion of biomarkers mandatory for upfront cancer prediction a likely response"
\end{abstract}

First draft submitted: 17 May 2019; Accepted for publication: 17 May 2019; Published online: 18 July 2019

Keywords: drug repurposing • financial resources $\bullet$ solid cancer $\bullet$ target therapy $\bullet$ therapeutic strategy

Cancer complexity has clearly emerged following basic and clinical research. However, despite the substantial prognostic improvement of newly diagnosed cancer patients, advanced disease often remains incurable. The molecular targeted therapies, companion and complementary diagnostic tests to select patients taking benefit from the new drugs, repurposing of drugs developed for other diseases and further approaches of research are hot issues of the last decades. Here, these issues are briefly examined and an insight for going beyond has been taken into account.

\section{The disappointing results of the molecular targeted therapies}

Over the last two decades several molecular targeted therapies have become available to treat cancer. Many of these drugs target gene derived proteins that are causally involved in driving cancer growth and progression. These therapies were expected to be more efficacious than existing treatments. Furthermore, as these new drugs target a specific protein or a small number of proteins, they were expected to cause fewer adverse side effects than conventional chemotherapy and radiotherapy - which have a more general mechanism of action. Therefore, so far, many resources have been spent for these new drugs. After two decades, it is appropriate that the success of these new molecular targeted therapies is evaluated. Indeed, many of the findings have been disappointing. Although hundreds of the novel drugs directed against specific targets have been evaluated in clinical trials, only a relatively small number have been approved for clinical use. With a few exceptions, the benefits of these drugs with respect to substantially improving overall survival has been marginal [1]. This limited benefit is either due to upfront intrinsic resistance or to the emergence of acquired resistance in those patients that initially respond $[2,3]$.

In an attempt to improve response to these new therapies, major effort has been devoted to the identification and validation of biomarkers that upfront predict likely benefit from the drug under consideration for administration. Indeed, the importance of having predictive biomarkers came to the fore recently when the NHS in the UK decided not to reimburse expensive drugs in the absence of biomarkers that accurately select patients likely to benefit. Furthermore, animate debate has occurred between the NHS's regulatory authorities and representatives of insurance companies following unfavorable cost-effectiveness ratio analysis with several different drugs including trastuzumab and bevacizumab $[4,5]$. The need has become urgent due to the extensive use of immune therapy and the programmed PD-1, PD-L1 inhibitors.

\section{New targeted therapies active on the immune system implement research for upfront predictive biomarkers}

Since 2010 immune-oncologic agents have been proposed for the cancer treatment; mainly melanoma, lung and urothelial cancers [6]. However, these drugs are commonly expensive, while the proportion of patients receiving 
benefit is limited. It derives a pressing need to select those patients for whom treatment is more likely to be beneficial. CDx and complementary diagnostic tests are expected to play a critical role in the selection of these patients and in the decision making process forming the basis of the so called precision medicine. CDx test is mandatory for the use of a specific drug while complementary diagnostic test can be used more largely with a class of agents. Expression of PD-1/PD-L1 and measurement of tumor infiltrating lymphocytes (TILs) evaluated by immune-histochemistry (IHC) as well as mismatch repair (MMR) assessed by genomic sequencing are among the most studied predictive biomarkers of response to immune oncology therapy. PD-1/PD-L1 expression on TILs predicts response to PD-1 blockade by PD-1/PD-L1 inhibitors and the burden of TILs is thought to reflect an immunological response to malignancy. However, a standardized method to evaluate the number of TILs is lacking. In the current clinical use, IHC approach to measure PD-L1 expression on tumor and immune cells is CDx for pembrolizumab in non-small-cell lung cancer patients. MMR predicts response to PD-1 blockade therefore is CDx for pembrolizumab in patients with solid tumors and high microsatellite instability. So far only pembrolizumab has been approved for non-small-cell lung cancer and for any solid tumor with the use of PD-L1 expression or MMR plus microsatellite instability assessment as CDx tests, respectively [6].

\section{New anticancer strategies: the simplest \& other more rational directions}

The lack of effectiveness in most unselected patients, and high treatment costs make the inclusion of biomarkers mandatory for upfront cancer prediction a likely response [6,7]. Also, this suggests to limit the resources for molecular targeted therapies research while proposing new anticancer approaches. Solid tumors account for most cancer related-deaths, and the history of molecular targeted therapies reflects our current inability to cure metastatic disease [8]. Despite molecular targeted therapies being overwhelmingly focused on the metastatic setting, most often advances were incremental and only in a minority of cases were the advances substantial. Among the new anticancer approaches, the repurposing of drugs developed for other diseases toward cancer should be mentioned as the most easily available [9] and probably with the highest positive cost benefit ratio. The rationale for repurposing stems from observations that different diseases share common molecular pathways and targets in the cell. As an example, the use of the antimalarial agents, chloroquine and hydroxychloroquine has been proposed for anticancer therapy. Thus, in experimental studies these drugs were shown to inhibit not only autophagy but also to affect the TLR9, p53 and CXCR4-CXCL12 pathway in cancer cells. Similarly, selective PDES5 inhibitors such as sildenafil, tadalafil and vardenafil, widely used in the treatment of erectile dysfunction and pulmonary arterial hypertension, are under active investigation as anticancer agents. In vitro and in vivo preclinical evidence has been reported on the ability of these compounds to induce apoptosis of cancer cells. Furthermore, these compounds were shown to mediate immune antitumor effects including reduction in regulatory $\mathrm{T}$ cells and/or myeloid-derived suppressor cells. Despite a lack of financial incentives for drug developers and limited drug development experience within the nonprofit sector, a Repurposing Drugs in Oncology Project that seeks to repurpose well-known and well-characterized noncancer drugs for new uses in oncology was launched [10]. In addition to having anticancer activity, some of these drugs undergoing evaluation for repurposing to cancer have been shown to sensitize tumor cells to conventional treatment. For example, sildenafil has been reported to reduce hypoxia and hence improving response to radiotherapy. However, to overcome the ongoing deadlock likely there is the need to look at the entire cancer complexity rather than to focus on single or few molecular pathways. Further promising investigations include interventions on more common general mechanisms. Examples are the relationship between tumor growth and immune evasion [11], the adoptive transfer of autologous lymphocytes specifically targeting proteins encoded by somatically mutated genes in metastatic patients [12] and the inhibition of cancer cell invasion and metastasization [13].

\section{Merits \& limits of the molecular targeted therapies: the way for going beyond}

During the last two decades, the great contribution of the targeted therapies research to the comprehension of the complexity of cancer by many elucidated pathological molecular pathways cannot be denied. Some of them were used to design the novel drugs and to stimulate new genetic and epigenetic studies in order to better understand the origin of the discovered molecular alterations [14]. In spite of this, the targeted therapies studies implied an important restraint to the research in other fields due to the relevant involved resources. The molecular-targeted therapy era unraveled the genetic instability (recurrent somatic mutations). Plasticity of the phenotype, polyclonal origin and continuous selection of subclones [15], redundancy (multiple molecular mechanisms addressing the same outcome) and the cancer cell cross-talk with the tumor microenvironment [16] are the main hurdles of the cancer system to be overcome. In the metastatic setting, they account for de novo and acquired resistance to any new 
drug that is effective in a small proportion of the target population and for a limited time. The repurposing of drugs developed for other diseases towards cancer is an easy and cheap answer to this cancer complexity. The other above-mentioned research show are novel more in-depth attempts to identify and efficaciously interfere with some crucial points of the cancer system. Namely, the early research hypothesized a close relationship of tumor burden with immune evasion and considered minimal residual disease as a favorable condition to impede clinically detectable metastases [11]. In another research, advanced technologies allowed immune recognition of somatic mutations in biopsies of metastatic lesions and the infusion of mutant-protein reactive TILs produced objective cancer regressions [12]. The later research has observed that anti-invasion and antimetastatic drugs are the missing category in the current armory of anticancer therapies. Actin polymerization and contractility are proposed as targets of a new category of drugs under investigation termed 'migrastatics', capable of interfering with all modes of cancer cell invasion and metastasis [13]. The postoperative monitoring of minimal residual disease by liquid biopsy is a further emerging field of research that can support the others. Enumeration of the circulating tumor cells [16], detection of plasma circulating tumor DNA (ct DNA) [8] and isolation from blood of cell-free DNA (cfDNA) [17] are different modalities. They all foster the hope of capturing tumor evolution to better drive precision medicine such as a better selection of patients to be treated in the adjuvant setting.

\section{Conclusion}

After two decades of research on and use of molecular targeted therapies for advanced cancer treatment, the results are disappointing due to limited benefit and high cost. Companion and complementary diagnostic tests are expected to play a critical role as predictive biomarkers in the selection of patients taking benefit from the new drugs and in the decision-making process forming the basis of the so called precision medicine. Repurposing of drugs developed for other diseases and other approaches more in-depth taking into account the cancer complexity have been proposed or have produced preliminary encouraging results. We feel that moving resources, at least in part, from research on target molecular therapy to implement the above-mentioned new types of investigations is worthwhile and can be helpful for progress in cancer treatment.

\section{Financial \& competing interests disclosure}

The authors have no relevant affiliations or financial involvement with any organization or entity with a financial interest in or financial conflict with the subject matter or materials discussed in the manuscript. This includes employment, consultancies, honoraria, stock ownership or options, expert testimony, grants or patents received or pending, or royalties.

No writing assistance was utilized in the production of this manuscript.

\section{References}

1. Bivona TG, Doebele RC. A framework for understanding and targeting residual disease in oncogene-driven solid cancers. Nat. Med. 22(5), 472-478 (2016).

2. Davis C, Naci H, Gurpinar E, Poplavska E, Pinto A, Aggarwal A. Availability of evidence of benefits on overall survival and quality of life of cancer drugs approved by European Medicines Agency: retrospective cohort study of drug approvals 2009-2013. BMJ 4(359), j4530 (2017).

3. Cohen D. Cancer drugs: high price, uncertain value. BMJ 4(359), j4543 (2017).

4. Barrett A, Roques T, Small M, Smith RD. How much will Herceptin really cost? BMJ 333(7578), 1118-1120 (2006).

5. D'Agostino RB. Changing end points in breast-cancer drug approval - the avastin story. NEJ M 2 365(2), e2 (2011).

6. Ferreira CG, Nicolini A, Dalurzo L, Stefani S, Teich V, Leighl N. The value of biomarkers in optimizing the use of immune-oncologic therapy. Curr. Drug Targets 20(1), 81-86 (2019).

7. Nicolini A, Ferrari P, Duffy MJ. Prognostic and predictive biomarkers in breast cancer: past, present and future. Semin. Cancer Biol. 52(Pt1), 56-73 (2018).

8. Dasari A, Grothey A, Kopetz S. Circulating tumor DNA-defined minimal residual disease in solid tumors: opportunities to accelerate the development of adjuvant therapies. J. Clin. Oncol. 36(35), 3437-3440 (2018).

9. Gupta SC, Sung B, Prasad S, Webb LJ, Aggarwal BB. Cancer drug discovery by repurposing: teaching new tricks to old dogs. Trends Pharmacol. Sci. 34(9), 508-517 (2013).

10. Pantziarka P, Bouche G, Meheus L, Sukhatme V, Sukhatme VP, Vikas P. The repurposing drugs in oncology (ReDO) project. Ecancermedicalscience 10(8), 442 (2014).

11. Nicolini A, Ferrari P, Rossi G, Carpi A. Tumour growth and immune evasionas targets for a new strategy in advanced cancer. Endocr. Relat. Cancer 25(11), R577-R604 (2018). 
12. Zacharakis $\mathrm{N}$, Chinnasamy $\mathrm{H}$, Black $\mathrm{M}$ et al. Immune recognition of somatic mutations leading to complete durable regression in metastatic breast cancer. Nat. Med. 24(6), 724-730 (2018).

13. Gandalovičová A, Rosel D, Fernandes $\mathrm{M}$ et al. Migrastatics-anti-metastatic and anti-invasion drugs: promises and challenges. Trends Cancer 3(6), 391-406 (2017).

14. Cancer Genome Atlas Network. Comprehensive molecular portraits of human breast tumours. Nature 490(7418), 61-70 (2012).

15. Soler A, Cayrefourcq L, Mazard T et al. Autologous cell lines from circulating colon cancer cells captured from sequential liquid biopsies as model to study therapy-driven tumor changes. Sci. Rep. 8(1), 15931 (2018).

16. Pantel K, Alix-Panabières C. Tumour microenvironment: informing on minimal residual disease in solid tumours. Nat. Rev. Clin. Oncol. 14(6), 325-326 (2017).

17. Lin SY, Orozco JIJ, Hoon DSB. Detection of minimal residual disease and its clinical applications in melanoma and breast cancer patients. Adv. Exp. Med. Biol. 1100, 83- 95 (2018). 